\title{
Application of Hemoadsorption in a Case of Liver Cirrhosis and Alcohol-Related Steatohepatitis with Preexisting Hepatitis C Infection
}

\author{
Stefan Büttner ${ }^{\mathrm{a}}$ Sammy Patyna ${ }^{\mathrm{a}}$ Benjamin Koch ${ }^{\mathrm{a}}$ Fabian Finkelmeier $^{\mathrm{b}}$ Helmut Geiger $^{\mathrm{a}}$ \\ Christoph Sarrazin $^{\mathrm{b}}$ Harald Farnik $^{\mathrm{b}}$

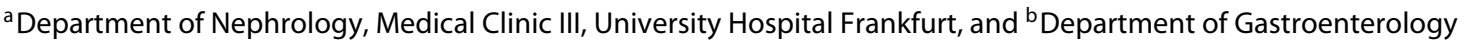 \\ and Hepatology, Medical Clinic I, University Hospital Frankfurt, Frankfurt, Germany
}

Dear Editor,

Acute-on-chronic liver failure, including decompensated alcoholic steatohepatitis (ASH), represents a distinct type of hepatic decompensation, accompanied by systemic inflammation, extrahepatic organ failure, and susceptibility to infection that can occur in patients with cirrhosis. Short-term mortality from decompensated alcohol-related liver disease is high with up to $10-20 \%$ at 1 month $[1,2]$. In most cases, corticosteroids are the only available treatment option; however, the proof for their efficacy is missing. Likewise, despite the availability of various techniques for liver organ support (i.e., MARS, SPAD), application is cumbersome and data on their clinical efficiency remain sparse [3]. We report the case of a 36-year-old male patient with a clinical history of chronic viral hepatitis $\mathrm{C}$ and longtime chronic alcohol abuse up to the point of admission to hospital and subsequently to ICU with decompensated ethanol toxic liver cirrhosis. At this point, the patient was hypotonic, tachycardic, and oliguric with upper gastrointestinal bleeding, with a MELD score of 40, and progressive hepatic encephalopathy. Initial attempts were made to stabilize the patient using albumin infusion and multiple paracenteses. However, in further course, he developed hepatorenal syndrome and subsequent dialysis dependency. Consequently, the patient was treated for more than a month in the intensive care unit to stabilize the cirrhosis and acute kidney injury. During this time, an evaluation as to whether the patient could be listed for a liver transplantation or not was rejected by the Liver Board. Since no transplant option existed, we continued therapy with available treatment options including steroid therapy with $40 \mathrm{mg}$ per day, however, with no significant improvement. Plasma bilirubin concentrations showed a significant increase of up to $24.5 \mathrm{mg} / \mathrm{dL}$; ammonia levels were $130 \mu \mathrm{g} / \mathrm{dL}$. In addition, transaminases (GOT $259 \mathrm{U} / \mathrm{L}$, GPT 59 $\mathrm{U} / \mathrm{L})$ as well as $\mu \mathrm{GT}$ (352 U/L) were markedly elevated and markers for spontaneous coagulation at this time were also poor. $\mathrm{He}$ also exhibited highly elevated markers of inflammation (leukocytes $43,000 / \mu \mathrm{L}$, C-reactive protein $3.46 \mathrm{mg} / \mathrm{dL}$, and interleukin-6 $42 \mathrm{pg} / \mathrm{mL}$ ). Continuous renal replacement therapy (CRRT) was started in the CVVHD mode (Multifiltrate, Fresenius Medical Care). During this phase, the patient received a low-dose norepinephrine infusion $(<0.025 \mu \mathrm{g} / \mathrm{kg} / \mathrm{min})$. As a "last resort" therapy, a hemoadsorp- tion treatment (Cytosorb; Cytosorbents $\mathrm{GmbH}$, Berlin Germany) was initiated with the rationale to remove inflammation-triggering factors and liver toxins (bile acids, bilirubin, ammonia) in the context of his systemic inflammatory condition as well as acute-on-chronic liver failure. In total, 2 treatments with CytoSorb were carried out running for $6 \mathrm{~h}$ each (blood flow rate $200 \mathrm{~mL} / \mathrm{min}$, heparin anticoagulation, predialyzer position), separated from one another by a treatment pause for 5 days to wait for the therapy effect due to non-existing evidence in this kind of patients. A significant reduction of bilirubin plasma levels was observed already within $4 \mathrm{~h}$ after the initiation of treatment (24.5 vs. $16.3 \mathrm{mg} / \mathrm{dL})$. Kinetic pre- and post-adsorber measurements during the second (hemoperfusion mode only without CVVHD) time of treatment confirmed efficient removal of ammonia, bilirubin, and bile acids (Fig. 1). During the first time of treatment, renal function and thus diuresis improved rapidly, so that CVVHD could be discontinued after the first treatment. After initially recovering with planned discharge to his home environment, the patient subsequently developed a nosocomial pneumonia, after

\section{KARGER}

(c) 2017 S. Karger AG, Basel 


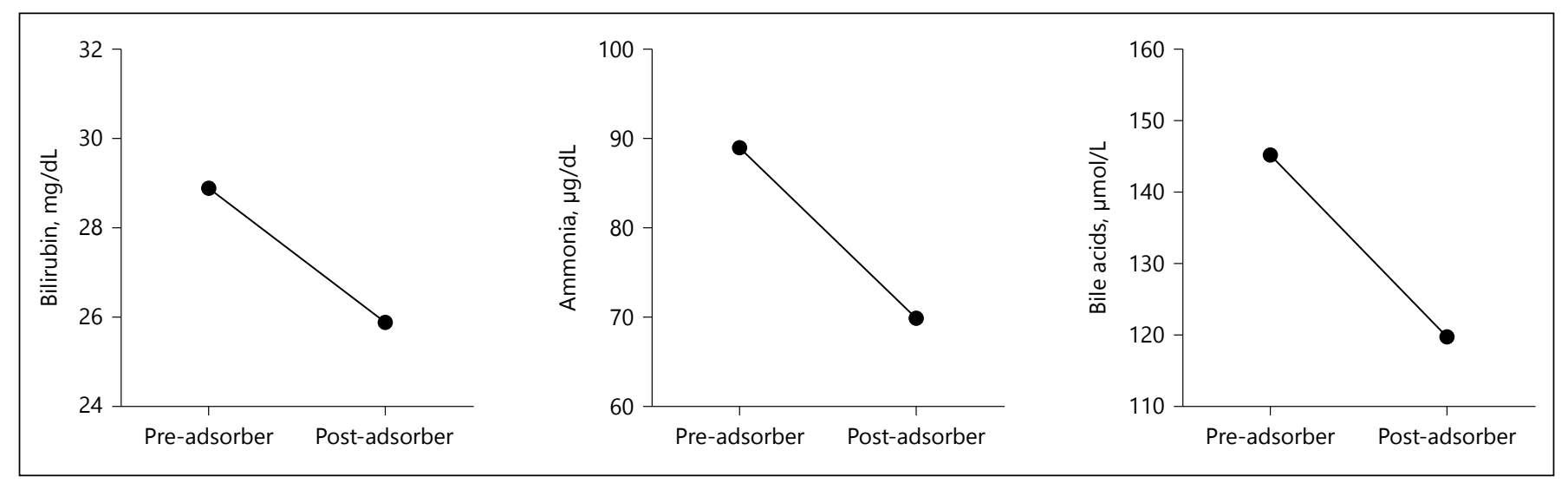

Fig. 1. Kinetic pre- and post-adsorber measurements during the second (hemoperfusion mode only without CVVHD) treatment showing an efficient removal of bilirubin, ammonia, and bile acids by the CytoSorb adsorber.

which the patient went into another episode of fulminant pneumogenic sepsis and died 3 weeks later on IMC. In this case, the treatment with a combination of CRRT and hemoadsorption using CytoSorb worked extremely well and effectively as liver support. As a consequence, hepatic encephalopathy improved significantly due to the efficient removal of liver toxins. In addition, measurement of pre- and post-adsorber values indicates that the removal of ammonia and bile acids is direct-

\section{References}

1 McPherson S, Lucey MR, Moriarty KJ: Decompensated alcohol related liver disease: acute management. BMJ 2016;352:1124.

2 Clària J, Stauber RE, Coenraad MJ, Moreau R, Jalan R, Pavesi M, Amorós À, Titos E, Alcaraz-Quiles J, Oettl K, Morales-Ruiz ly attributable to the adsorber. Moreover, the installation of the absorber into the CVVH circuit and the application of CytoSorb itself were easy and safe when compared to other procedures. Therefore, CytoSorb might represent a potential treatment option for patients with ASH and may be effective in young patients with severe inflammatory response in the context of ASH. However, further studies are necessary to evaluate the true benefits in this indication.

\section{Statement of Ethics}

Authors have declared that this study was performed in accordance with the research ethical guidelines.

\section{Disclosure Statement}

The other authors have no conflicts of interest associated with this report. There was no financial support for this study.
M, Angeli P, Domenicali M, Alessandria C, Gerbes A, Wendon J, Nevens F, Trebicka J, Laleman W, Saliba F, Welzel TM, Albillos A, Gustot T, Benten D, Durand $\mathrm{F}$, Ginès $\mathrm{P}$, Bernardi $\mathrm{M}$, Arroyo $\mathrm{V}$ : Systemic inflammation in decompensated cirrhosis: characterization and role in acuteon-chronic liver failure. Hepatology 2016; 64:1249-1264.

3 Parés A, Mas A: Extracorporeal liver support in severe alcoholic hepatitis. World J Gastroenterol 2014;20:8011-8017. 